\title{
It is safe to use transdermal glyceryl trinitrate to lower blood pressure in patients with acute ischaemic stroke with carotid stenosis
}

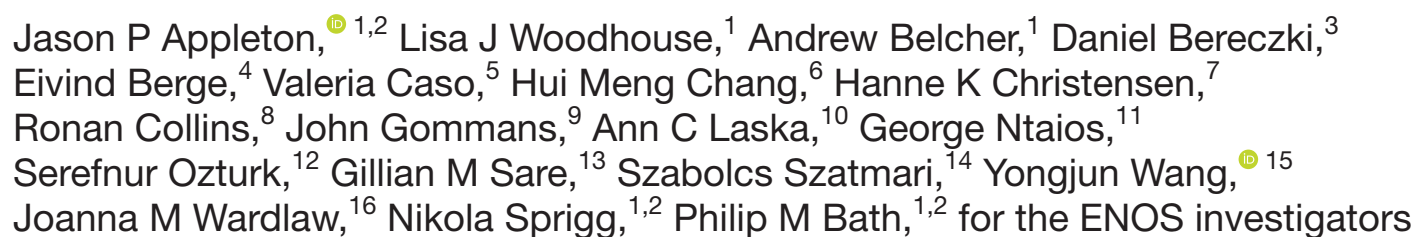

\section{ABSTRACT}

Background There is concern that blood pressure (BP) lowering in acute stroke may compromise cerebral perfusion and worsen outcome in the presence of carotid stenosis. We assessed the effect of glyceryl trinitrate (GTN) in patients with carotid stenosis using data from the Efficacy of Nitric 0xide in Stroke (ENOS) Trial.

Methods ENOS randomised 4011 patients with acute stroke and raised systolic BP $(140-220 \mathrm{~mm} \mathrm{Hg})$ to transdermal GTN or no GTN within 48 hours of onset. Those on prestroke antihypertensives were also randomised to stop or continue their medication for 7 days. The primary outcome was the modified Rankin Scale (mRS) at day 90. Ipsilateral carotid stenosis was split: $<30 \%$; $30-<50 \%$; $50-<70 \% ; \geq 70 \%$. Data are ORs with $95 \%$ Cls adjusted for baseline prognostic factors.

Results 2023 (60.5\%) ischaemic stroke participants had carotid imaging. As compared with $<30 \%, \geq 70 \%$ ipsilateral stenosis was associated with an unfavourable shift in $\mathrm{mRS}$ (worse outcome) at 90 days (OR $1.88,95 \% \mathrm{Cl}$ 1.44 to $2.44, p<0.001)$. Those with $\geq 70 \%$ stenosis who received GTN versus no GTN had a favourable shift in $\mathrm{mRS}$ (OR $0.56,95 \% \mathrm{Cl} 0.34$ to $0.93, \mathrm{p}=0.024$ ). In those with $50-<70 \%$ stenosis, continuing versus stopping prestroke antihypertensives was associated with worse disability, mood, quality of life and cognition at 90 days. Clinical outcomes did not differ across bilateral stenosis groups. Conclusions Following ischaemic stroke, severe ipsilateral carotid stenosis is associated with worse functional outcome at 90 days. GTN appears safe in ipsilateral or bilateral carotid stenosis, and might improve outcome in severe ipsilateral carotid stenosis.

\section{INTRODUCTION}

Blood pressure (BP) is elevated in $75 \%$ of patients presenting with acute ischaemic stroke $^{1}$ and is associated independently with poor clinical outcomes. ${ }^{23}$ Lowering elevated BP appears safe in acute ischaemic stroke, but has failed to show clinical benefit. ${ }^{4}$ There is a specific concern regarding BP lowering in the $15 \%$ of patients with significant carotid stenosis in whom cerebral perfusion may be compromised and where reducing BP might extend the ischaemic core and potentially worsen outcome. ${ }^{5}$ Data on BP reduction in severe ipsilateral or bilateral carotid stenosis are limited, although a meta-analysis found that lower BP was associated with an increased rate of stroke recurrence in bilateral carotid stenosis. ${ }^{6}$ In the ultra-acute prehospital and acute hospital situation, information on carotid stenosis is often not available and it is unclear whether BP lowering is safe in this group of patients with stroke.

The Efficacy of Nitric Oxide in Stroke (ENOS) Trial assessed the safety and efficacy of transdermal glyceryl trinitrate (GTN) and of continuing prestroke antihypertensives in 4011 patients with acute stroke. ${ }^{7}$ Although GTN lowered BP by $7 / 3.5 \mathrm{~mm} \mathrm{Hg}$ at day 1 , GTN did not influence functional outcome at 90 days. $^{7}$ However, when administered within 6 hours of stroke onset GTN improved several clinical outcomes. ${ }^{8}$ The aim of the current preplanned substudy ${ }^{9}$ was to assess the safety and efficacy of BP lowering on clinical outcomes in patients with acute ischaemic stroke and carotid stenosis.

\section{METHODS}

Details pertaining to the ENOS trial protocol, statistical analysis plan, baseline characteristics and main trial results have been published. ${ }^{810-12}$ In summary, the ENOS Trial recruited 4011 patients with acute stroke within 48 hours of onset with high systolic BP (140-220 $\mathrm{mm} \mathrm{Hg}$ ) and randomised them to GTN $5 \mathrm{mg}$ patch or no patch for 7 days. Those participants taking antihypertensive medication prior to their index event were also randomised to continue or stop these 
drugs for 7 days. Known carotid stenosis was not an exclusion criterion. Written consent to participate was given by patients or relatives/carers in those who lacked capacity. The ENOS Trial was registered (ISRCTN99414122).

\section{Patient and public involvement}

Patients and public were not involved in the development of this preplanned secondary analysis of the ENOS trial.

\section{Carotid stenosis}

Clinical information on carotid stenosis was collected by investigators during the participant's index event admission. Clinical imaging using either carotid Doppler, MR angiography or CT angiography was performed as per local protocol. Investigators entered the $\%$ of stenosis of both left and right internal carotid arteries using North American Symptomatic Carotid Endarterectomy Trial (NASCET) criteria where available (online supplementary material).$^{13}$ Data were checked and validated but no central adjudication of carotid imaging was performed.

Participants who had a final diagnosis of ischaemic stroke and who had carotid data available were included in this substudy. Grades of carotid stenosis were defined as follows:

- Unilateral carotid stenosis ipsilateral to the symptomatic hemisphere: ${ }^{13}<30 \%, 30-<50 \%, 50-<70 \%$, $\geq 70 \%$.

- Bilateral carotid stenosis (\% for both carotid arteries): $<30 \%, 30-<50 \%, \geq 50 \%$.

\section{Haemodynamic measures}

$\mathrm{BP}$ and heart rate were measured peripherally at baseline (three measurements) and on days 1-7 (two measurements/day), using validated automated equipment (Omron $705 \mathrm{CP}){ }^{14}$

\section{Clinical outcomes}

The primary outcome in ENOS was functional outcome measured using the modified Rankin Scale ${ }^{15}$ (mRS, a seven-level categorical scale where $0=$ independentand $6=$ dead) at 90 days. Day 90 secondary outcomes included disability (Barthel Index), ${ }^{16}$ mood (Zung Depression Scale), ${ }^{17}$ quality of life (health utility status calculated using the European Quality of Life 5-dimensions three levels version, and Visual Analogue Scale) ${ }^{18}$ and cognition (telephone mini-mental state examination, ${ }^{19}$ modified Telephone Interview for Cognition Scale [TICS-M] ${ }^{20}$ and verbal fluency). Patients who had died by day 90 were assigned a worst score for the outcomes. Safety data were collected on all-cause mortality at day 90 , early neurological deterioration (a minimum 5-point reduction overall or $>2$ points reduction in the consciousness domain from baseline to day 7 on the Scandinavian Stroke Scale [SSS]), symptomatic hypotension, hypertension or headache by day 7. The National Institute of Health Stroke Scale (NIHSS) was calculated from SSS. ${ }^{21}$ Day 90 outcomes were recorded by trained blinded assessors via telephone at national coordinating centres.

\section{Statistical analysis}

In line with the ENOS Trial statistical analysis plan and statistical analyses performed in the primary publication, intention-to-treat analysis of data was carried out. ${ }^{11}$ Data are number (\%), median [IQR] or mean (SD). Baseline characteristics between grades of carotid stenoses were assessed using the $\chi^{2}$ test for categorical variables and one-way analysis of variance for continuous variables.

Associations between carotid stenosis grades and outcomes were assessed using multiple linear regression, ordinal logistic regression or binary logistic regression with adjustment for baseline prognostic covariates including age, sex, baseline mRS score, history of previous stroke, history of diabetes mellitus, total anterior circulation stroke, nitrate use, baseline SSS, thrombolysis, feeding status, time to randomisation and baseline systolic BP. Analyses involving the whole population were also adjusted for treatment allocation. Associations between BP change from baseline to day 1 and outcome across degrees of carotid stenosis were assessed per $10 \mathrm{~mm} \mathrm{Hg}$ reduction in BP. Interaction $\mathrm{p}$ values were obtained by adding an interaction term to statistical models. Data are mean difference or OR and associated $95 \%$ CIs, with significance defined as $\mathrm{p} \leq 0.05$. Analyses were performed using SPSS V.24 (Chicago, Illinois, USA).

\section{RESULTS}

Of 4011 participants, 2023 (50.4\%) had a final diagnosis of ischaemic stroke and carotid imaging data (GTN 1002 vs no GTN 1021, table 1). One thousand three hundred and nineteen $(32.9 \%)$ patients with ischaemic stroke did not have carotid imaging, typically those with more severe stroke (carotid imaging: SSS 36.6 [12.4], no imaging: SSS 30.6 [13.7], $\mathrm{p}<0.001$ ); there was no relationship between country of enrolment and whether carotid imaging was performed (data not shown). Of 2023 participants with carotid data, 148 (7.3\%) had 50-<70\% ipsilateral stenosis, $213(10.5 \%)$ had $\geq 70 \%$ ipsilateral stenosis and 97 (4.8\%) had $\geq 50 \%$ bilateral stenosis. Age and rates of treated hypertension, diabetes mellitus, atrial fibrillation, history of transient ischaemic attack, ischaemic heart disease and peripheral arterial disease differed across grades of ipsilateral carotid stenosis. Those with higher degrees of ipsilateral carotid stenosis were more likely to be male, current smokers, have more severe strokes with higher NIHSS and lower Glasgow Coma Scale Scores, fewer cardioembolic and small vessel disease-related strokes, and more received thrombolysis treatment (table 1). As compared with patients with carotid imaging data, those without had a worse functional outcome at day 90: mRS 4 [3] versus 3 [3], (OR 1.76, 95\% CI 1.54 to 2.01, $\mathrm{p}<0.001$ ).

\section{Relationship between carotid stenosis and outcome}

Across all patients and as compared with participants with $<30 \%$ ipsilateral stenosis, those with $\geq 70 \%$ stenosis 


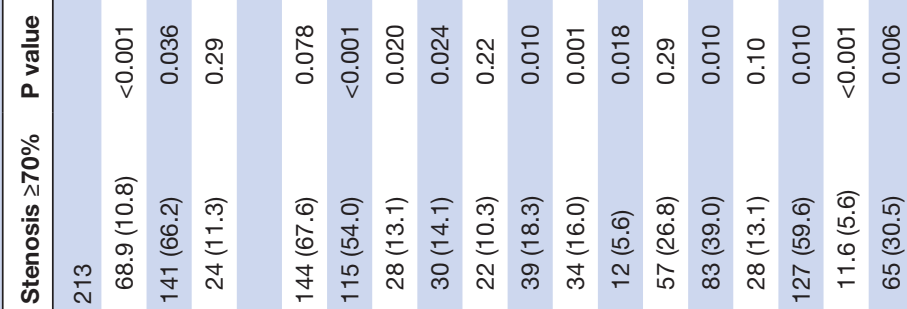

产

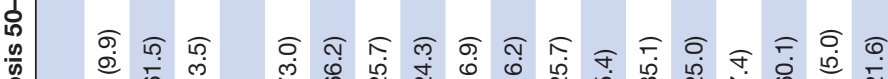

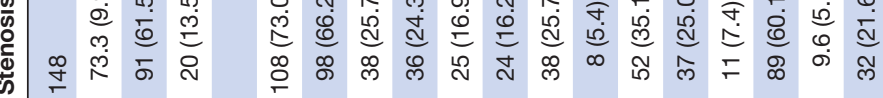

今ें.

$\frac{\pi}{20}$

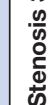

की

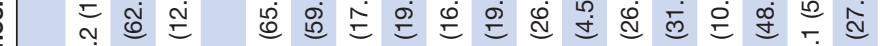
离

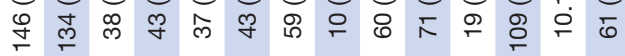

๖े

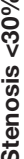

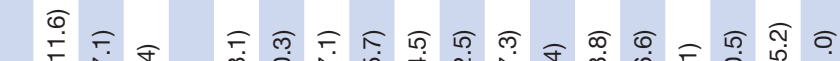

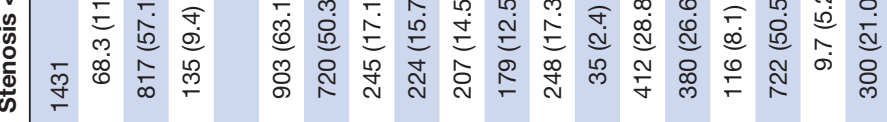

을

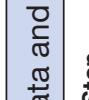

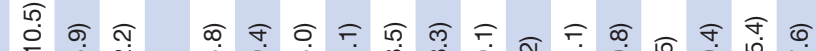

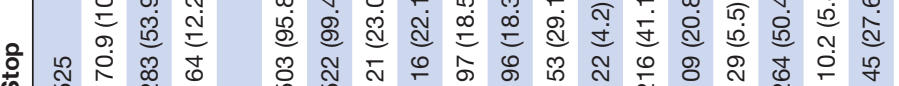

西

을

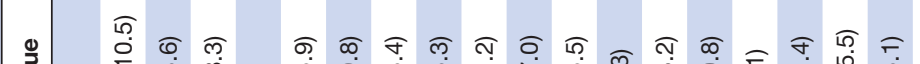

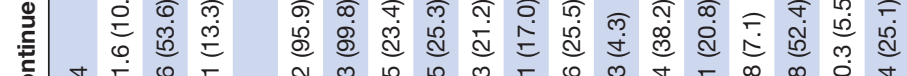

o

章

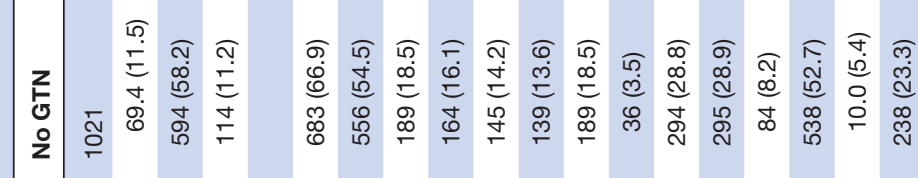

$\frac{\pi}{0}$

(1)

.

产

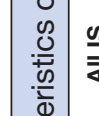

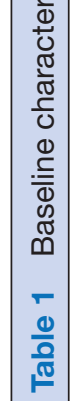

गु

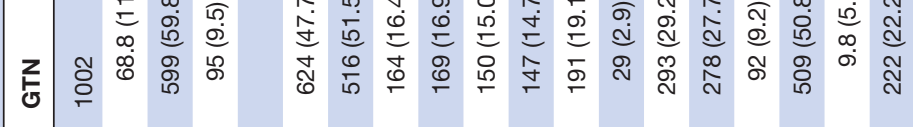

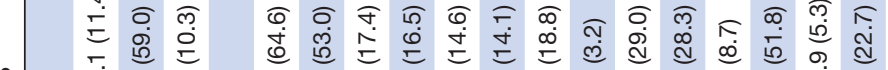

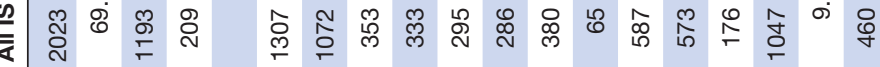

离

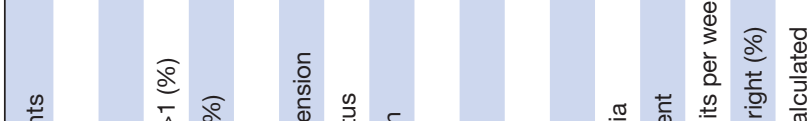

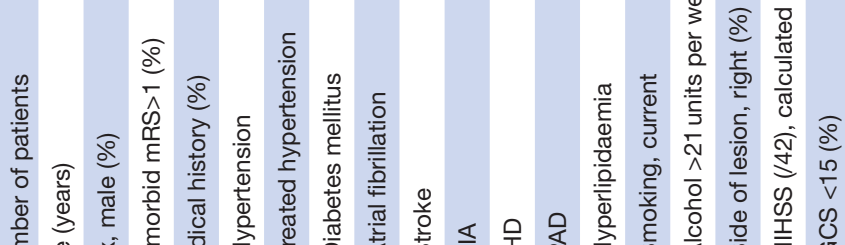

कิ

สุ

仓

辛

ণุ

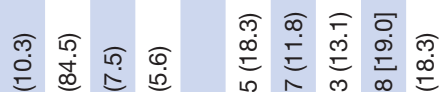

ก

ஸि

ल $\bar{\sigma}$ क

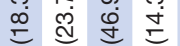

于

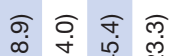

$\stackrel{\substack{c \\ \infty}}{\infty}$

ล

ल $\widehat{\infty} \widehat{0}$

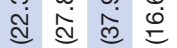

당

ब ธุ

过 守

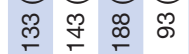

बิ क़

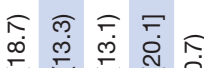

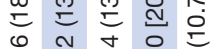

i $\infty \begin{aligned} & \infty \\ & i\end{aligned}$

ल. $\widehat{\infty} \bar{\infty}$

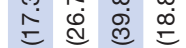

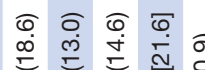

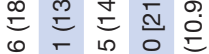

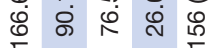

ㄱำ

ه.

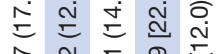

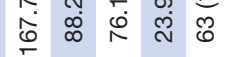

牙 过

ए

$\checkmark$ एᄂ

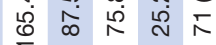

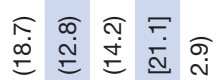

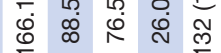

กิ

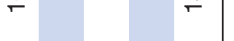

@)

$\sum_{\substack{0 \\ 0}}^{2}$

음

$\overrightarrow{\vec{F}}$

음

$\frac{\overline{0}}{\mathbb{\Phi}}$

क

○

$\overrightarrow{\vec{\omega}}$

క

옹

ठ

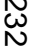

일

$\overrightarrow{0}$

常

옴

6

$\sum^{\circ}$

Oे

$\stackrel{8}{\circ}$

옥

西

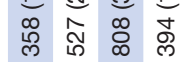

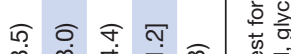

$\infty 0$

$\underset{x}{x}$

동

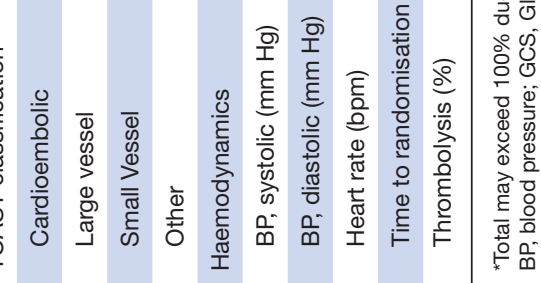


had an unfavourable shift in mRS (worse outcome) at day 90 (OR $1.88,95 \%$ CI 1.44 to 2.44 , p<0.001, table 2 , figure 1); significant associations with worse disability and quality of life, more depression and poorer cognitive scores were also seen. In addition, those with $\geq 70 \%$ stenosis had an increased rate of recurrent ischaemic stroke, clinical deterioration, neurological deterioration, and higher NIHSS Scores at day 7 (online supplementary table 1).

\section{Effects of GTN versus no GTN}

Those with $\geq 70 \%$ ipsilateral stenosis who were randomised to GTN had a significant shift in $\mathrm{mRS}$ to less death or dependency at 90 days (OR 0.56, 95\% CI 0.34 to $0.93, \mathrm{p}=0.024$ ) (table 3 , figure 2). However, GTN did not influence mRS across the other carotid stenosis groups, although there were higher cognitive scores at day 90 in those with $50-<70 \%$ stenosis but not in other stenosis groups. Headache, a recognised side effect of GTN, was more common in those with $\geq 70 \%$ stenosis who were randomised to GTN; non-significant increases in headache with GTN were also reported in the other carotid stenosis groups (online supplementary table 2).

\section{Effects of continuing versus stopping prestroke antihypertensives}

In those with 50-<70\% ipsilateral stenosis, continuing prestroke antihypertensives was associated with more depression, worse disability and quality of life, and poorer cognitive score (TICS-M) at day 90 compared with stopping prestroke antihypertensives, independent of GTN allocation. No effect on mRS was seen nor were these effects replicated in those with more severe $(\geq 70 \%)$ ipsilateral stenosis (table 3 ).

\section{Bilateral carotid stenosis}

Only $97 / 2023$ (4.8\%) had $\geq 50 \%$ bilateral carotid stenosis. There were no significant associations across degrees of bilateral carotid stenosis with clinical outcome measures at either 7 or 90 days (online supplementary table 4). Neither GTN nor continuing prestroke antihypertensives influenced outcome in participants with bilateral carotid stenosis (data not shown).

\section{BP change and carotid stenosis}

The largest fall in BP was seen from baseline to day 1 in those randomised to GTN versus no GTN $(7 / 3 \mathrm{~mm} \mathrm{Hg}$ overall) and did not significantly differ across degrees of ipsilateral (online supplementary table 2, interaction $\mathrm{p}=0.22$ ) or bilateral carotid stenosis (interaction $\mathrm{p}=0.19$ ). When assessed across degrees of ipsilateral carotid stenosis, change in BP from baseline to day 1 did not influence mRS at day 90 (online supplementary table 5). There were no significant interactions between treatment with GTN or continuing prestroke antihypertensives in relation to $\mathrm{BP}$ change and outcome. Similarly, across degrees of bilateral carotid stenosis, change in BP from

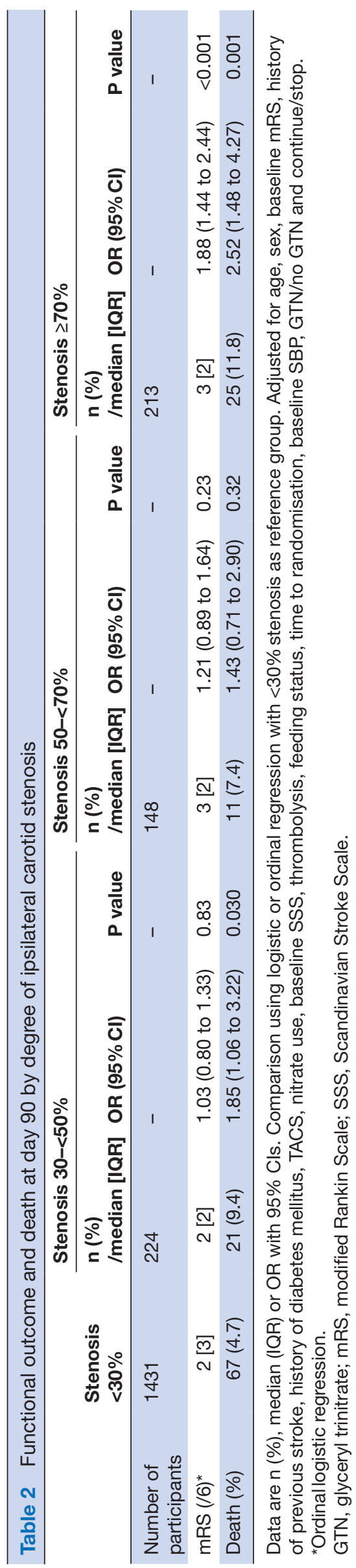




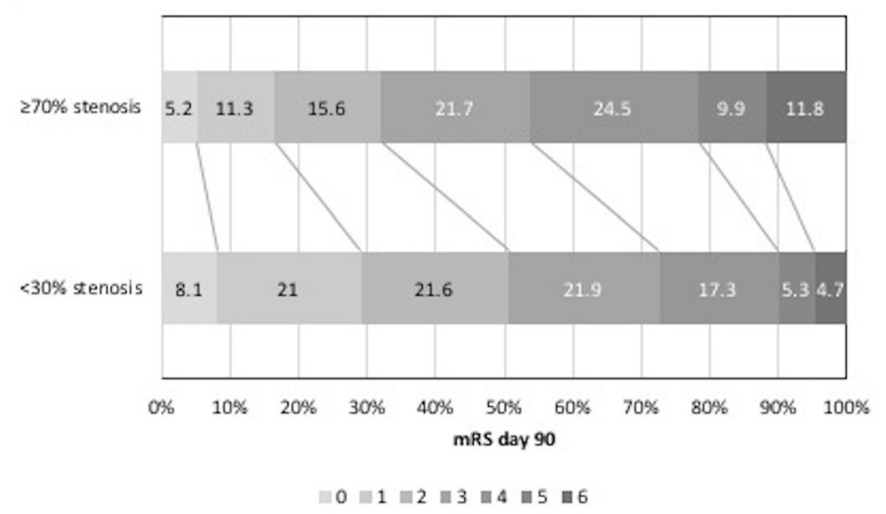

Figure $1 \mathrm{mRS}$ at day $90<30 \%$ vs $\geq 70 \%$ ipsilateral stenosis. mRS, modified Rankin Scale.

baseline to day 1 did not influence mRS at day 90 (online supplementary table 6).

\section{DISCUSSION}

In this preplanned ENOS substudy, ${ }^{9}$ taking all patients irrespective of treatment allocation, the presence of severe $(\geq 70 \%)$ ipsilateral symptomatic carotid stenosis was associated with unfavourable clinical outcomes both early and late after acute stroke. However, treatment with GTN versus no GTN was associated with a significant shift to less death or dependency at 90 days in those participants with $\geq 70 \%$ ipsilateral stenosis. Continuing versus stopping prestroke antihypertensives was associated with worse secondary outcomes in those with $50-<70 \%$ ipsilateral stenosis. Modest BP lowering was safe in patients with acute stroke in the context of unilateral and bilateral carotid stenosis.

It has long been established that higher degrees of symptomatic carotid stenosis are associated with early stroke recurrence and subsequent dependency after minor stroke and transient ischaemic attack; carotid endarterectomy is therefore recommended. ${ }^{22}$ This dataset suggests that patients with more severe strokes with large artery disease who may not have been eligible for carotid revascularisation have both poor early and late clinical outcomes as expected. These findings are likely to be even stronger in clinical practice as the $32.9 \%$ patients with ischaemic stroke who did not have carotid imaging had more severe strokes and therefore worse clinical outcome than those with imaging.

Although pathophysiological data have demonstrated dysfunctional cerebral autoregulation in severe carotid stenosis, ${ }^{23-25}$ and cerebral blood flow can become dependent on systemic $\mathrm{BP},{ }^{5}$ there are limited prospective data assessing BP lowering in acute stroke with carotid stenosis. Previous blinded analysis of ENOS performed during recruitment revealed that $\mathrm{BP}$ lowering in the context of carotid stenosis was safe. ${ }^{9}$ We have reinforced that finding here with no evidence to suggest modest BP lowering

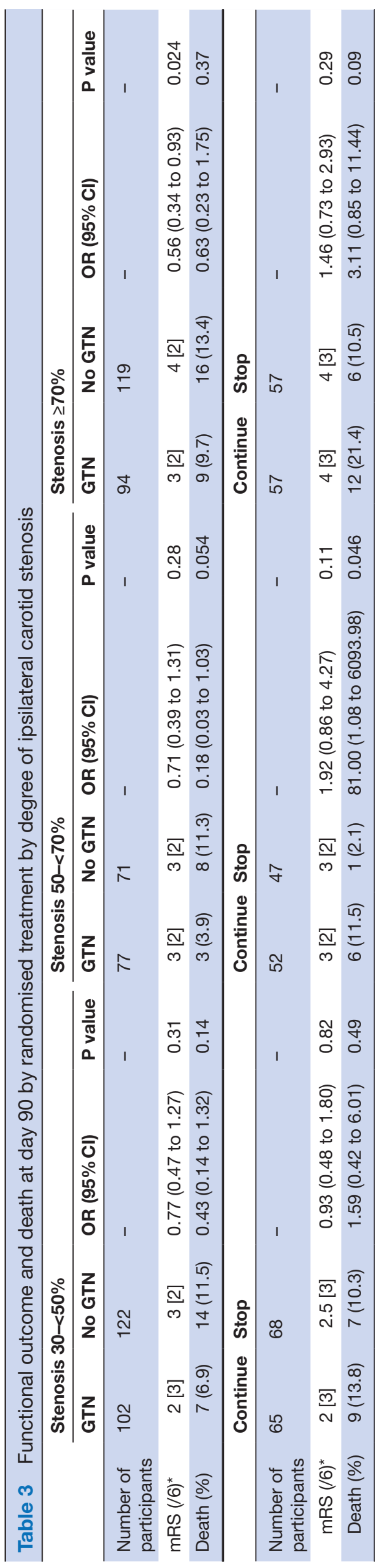




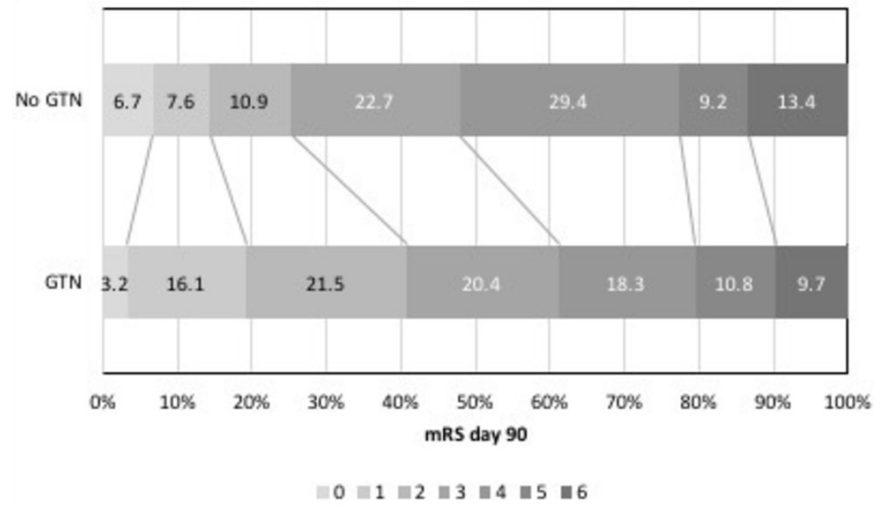

Figure $2 \mathrm{mRS}$ at day 90 in those with $\geq 70 \%$ ipsilateral stenosis GTN versus no GTN. GTN, glyceryl trinitrate; mRS, modified Rankin Scale.

is associated with stroke recurrence or other poor outcomes. A post hoc analysis of the Scandinavian Candesartan Acute Stroke Trial (SCAST) found no clear evidence to suggest that BP lowering was detrimental in patients with carotid stenosis, but there were non-significant tendencies towards increased stroke progression and poor functional outcome with candesartan. ${ }^{26}$ As in the present substudy, the observed BP lowering effect in SCAST was modest $(5 / 2 \mathrm{~mm} \mathrm{Hg})$ and therefore differing drug class effects may explain the differences observed between the present analysis and SCAST. Further, continuing prestroke antihypertensives in those with modest ipsilateral carotid stenosis $(50-<70 \%)$ was associated with poorer outcomes across several secondary domains, but not in those with $\geq 70 \%$ ipsilateral stenosis. This may be due to those with modest stenosis having greater baseline comorbidity (higher mRS, more hypertension, diabetes, previous stroke, ischaemic heart disease and hyperlipidaemia) than those with $\geq 70 \%$ stenosis. This imbalance may represent selection bias, whereby patients with more severe stroke and severe stenosis were not imaged as doing so would not change management. It is unclear whether there are drug class-specific mechanisms that may be harmful in the context of carotid stenosis early after stroke, but we add to evidence from the main ENOS Trial that routinely continuing prestroke antihypertensives should perhaps be avoided until the patient is neurologically stable. ${ }^{7}$ Whether larger precipitous drops in BP are safe is beyond the scope of this substudy, but given the uncertainty this practice should perhaps be avoided.

The shift in mRS to less death or dependency with GTN seen in those with $\geq 70 \%$ ipsilateral carotid stenosis may be related to effects other than BP lowering. As a vasodilator, GTN did not reduce cerebral blood flow in patients with acute stroke despite lowering peripheral and central BP. ${ }^{27} 28$ Although it is unclear whether GTN improves cerebral blood flow, ${ }^{28}$ it can be hypothesised that GTN may improve collateral blood supply via surface pial collaterals ${ }^{29}$ and thereby maintain blood flow to the ischaemic penumbra in the context of carotid stenosis when given early. A similar potential beneficial effect of transdermal GTN administered within 4 hours of stroke onset in patients with $\geq 70 \%$ ipsilateral carotid stenosis was seen in a preliminary analysis of 314 patients from the Rapid Intervention with Glyceryl trinitrate in Hypertensive stroke Trial-2 (RIGHT-2). ${ }^{30}$

Bilateral carotid stenosis is uncommon $(4.8 \%$ in this analysis) and therefore data regarding the safety of BP lowering in patients with acute stroke in this context are scanty. ${ }^{6}$ Impaired cerebral perfusion is more common in this population than in unilateral carotid stenosis and although we found no evidence that modest BP lowering was unsafe, further data are required to address this question.

This study represents the largest trial-based analysis of BP lowering in patients with acute ischaemic stroke with carotid stenosis to date. However, there are limitations. First, not all patients with ischaemic stroke had carotid imaging studies performed, a deficiency most prominent in patients with severe stroke who would not be appropriate candidates for revascularisation therapy. Second, the analyses across degrees of carotid stenosis are subgroup analyses and, so the results may represent chance. Third, the median time to randomisation in ENOS was 26 hours and so the effect of BP lowering in the context of carotid stenosis within the first few hours of ischaemic stroke remains unclear. Fourth, no adjustment was made for multiplicity of testing due to the exploratory nature of the study. Fifth, imaging information on carotid stenosis was provided by investigators at the site with unknown reporting criteria (NASCET, ${ }^{13}$ European Carotid Surgery Trial [ECST] $),{ }^{31}$ and Carotid and Vertebral Artery Transluminal Angioplasty Study (CAVATAS) ${ }^{32}$ ) and were not centrally adjudicated. However, data were validated and checked for accuracy. Sixth, it was not possible to adjust cognitive outcome data for cognition at baseline and many of the differences seen at 3 months in association with degrees of stenosis (apart from allocated trial treatment differences) likely reflect baseline status. Seventh, ENOS assessed mild to moderate BP lowering and the results presented here do not provide information on the effects of intensive BP lowering. Last, data on carotid endarterectomy were not captured prospectively in ENOS and were therefore unavailable for the population studied, although in a population of patients with mostly moderate-to-severe stroke it is unlikely that many patients underwent endarterectomy.

In summary, this ENOS substudy has demonstrated that severe ipsilateral carotid stenosis is associated with worse clinical outcomes at 7 days and 90 days after acute stroke irrespective of treatment allocation. Transdermal GTN improved functional outcome at 90 days in those with $\geq 70 \%$ ipsilateral stenosis and was safe across all degrees of 
carotid stenosis whether unilateral or bilateral. Further, modest BP lowering with GTN was safe in the context of carotid stenosis although continuing prestroke antihypertensives was associated with poorer secondary outcomes in those with $50-<70 \%$ ipsilateral carotid stenosis. Future studies should establish whether GTN and other BP lowering therapies have specific mechanistic properties that may be of benefit in acute stroke in the context of carotid stenosis.

\section{Author affiliations}

${ }^{1}$ Stroke, Division of Clinical Neurosciences, University of Nottingham, Nottingham, UK

${ }^{2}$ Stroke, Nottingham University Hospitals NHS Trust, Nottingham, UK

${ }^{3}$ Department of Neurology, Semmelweis University, Budapest, Hungary

${ }^{4}$ Department of Internal Medicine and Cardiology, Oslo University Hospital, Oslo, Norway

${ }^{5}$ Stroke Unit, Santa Maria della Misericordia Hospital, University of Perugia, Perugia, Italy

${ }^{6}$ Department of Neurology, Singapore General Hospital, Singapore, Singapore

${ }^{7}$ Neurology, Bispebjerg and Frederiksberg Hospital, Copenhagen, Denmark

${ }^{8}$ Tallaght Hospital, Trinity College Dublin, Dublin, Ireland

${ }^{9}$ Department of Medicine, Hawke's Bay District Health Board, Hastings, New Zealand

${ }^{10}$ Department of Clinical Science, Danderyd Hospital, Karolinska Institute, Stockholm, Sweden

${ }^{11}$ Department of Medicine, University of Thessaly, Larissa, Greece

${ }^{12}$ Neurology, Selcuk University Faculty of Medicine, Konya, Turkey

${ }^{13}$ Neurology, Nottingham University Hospitals NHS Trust, Nottingham, UK

${ }^{14}$ Department of Neurology, Clinical County Emergency Hospital, Targu Mures,

Romania

${ }^{15}$ Neurology, Beijing Tiantan Hospital, Beijing, China

${ }^{16}$ Centre for Clinical Brain Sciences, Edinburgh, UK

Acknowledgements The authors thank the participants and investigators who took part in the ENOS trial.

Collaborators ENOS investigators' details are specified in the online supplementary file.

Contributors JPA performed the analyses and wrote the first draft. PMB conceived the study and is corresponding author and project guarantor. LJW is the trial statistician. DB, EB, VC, HMC, HKC, RC, JG, ACL, GN, SO, GMS, NS, SS, YW, JW are members of the trial steering/international advisory committee. LJW, AB, DB, EB, VC, HMC, HKC, RC, JG, ACL, GN, SO, GMS, SS, YW, JW, NS commented upon and approved the manuscript for publication.

Funding ENOS was funded by Bupa Foundation and the Medical Research Council (G0501797). JPA is funded by NIHR TARDIS (10/104/24) and BHF RIGHT-2 (CS/14/4/30972).

Competing interests PMB is Stroke Association Professor of Stroke Medicine, Chief Investigator of MRC ENOS, and a NIHR Senior Investigator. PMB reports grants from British Heart Foundation and the NIHR Health Technology Assessment Programme, during the conduct of the study; other from Platelet Solutions Ltd, personal fees from Diamedica, personal fees from Nestle, personal fees from Phagenesis Ltd, personal fees from ReNeuron, personal fees from Athersys, personal fees from Covidien, outside the submitted work.

Patient consent for publication Not required.

Ethics approval The ENOS Trial was approved by national and local ethics committees and competent authorities in participating countries.

Provenance and peer review Not commissioned; internally peer reviewed. Data sharing statement Supplementary material are available online.

Open access This is an open access article distributed in accordance with the Creative Commons Attribution 4.0 Unported (CC BY 4.0) license, which permits others to copy, redistribute, remix, transform and build upon this work for any purpose, provided the original work is properly cited, a link to the licence is given, and indication of whether changes were made. See: https://creativecommons.org/ licenses/by/4.0/.

\section{REFERENCES}

1. Oppenheimer S, Hachinski V. Complications of acute stroke. Lancet 1992;339:721-4.

2. Leonardi-Bee J, Bath PM, Phillips SJ, et al. Blood pressure and clinical outcomes in the International Stroke Trial. Stroke 2002;33:1315-20.

3. Vemmos KN, Tsivgoulis G, Spengos K, et al. U-shaped relationship between mortality and admission blood pressure in patients with acute stroke. J Intern Med 2004;255:257-65.

4. Bath PM, Krishnan K. Interventions for deliberately altering blood pressure in acute stroke. Cochrane Database Syst Rev 2014(10):CD000039.

5. Willmot M, Leonardi-Bee J, Bath PM. High blood pressure in acute stroke and subsequent outcome: a systematic review. Hypertension 2004;43:18-24.

6. Rothwell PM, Howard SC, Spence JD. Relationship between blood pressure and stroke risk in patients with symptomatic carotid occlusive disease. Stroke 2003;34:2583-90.

7. Bath P, Woodhouse L, Scutt P, et al. Efficacy of nitric oxide, with or without continuing antihypertensive treatment, for management of high blood pressure in acute stroke (ENOS): a partial-factorial randomised controlled trial. Lancet 2015;385:617-28.

8. Woodhouse L, Scutt P, Krishnan K, et al. Effect of Hyperacute Administration (Within 6 Hours) of Transdermal Glyceryl Trinitrate, a Nitric Oxide Donor, on Outcome After Stroke: Subgroup Analysis of the Efficacy of Nitric Oxide in Stroke (ENOS) Trial. Stroke 2015;46:3194-201.

9. Sare GM, Geeganage C, Bath PM. High blood pressure in acute ischaemic stroke-broadening therapeutic horizons. Cerebrovasc Dis 2009;27:156-61.

10. ENOS Trial Investigators. Glyceryl trinitrate vs. control, and continuing vs. stopping temporarily prior antihypertensive therapy, in acute stroke: rationale and design of the Efficacy of Nitric Oxide in Stroke (ENOS) trial (ISRCTN99414122). Int J Stroke 2006;1:245-9.

11. Bath PM, Brainin M, Brown C, et al. Testing devices for the prevention and treatment of stroke and its complications. Int J Stroke 2014;9:683-95.

12. ENOS Investigators. Baseline characteristics of the 4011 patients recruited into the 'Efficacy of Nitric Oxide in Stroke' (ENOS) trial. Int $J$ Stroke 2014;9:711-20.

13. Barnett HJM, Taylor DW, Haynes RB, et al. Beneficial effect of carotid endarterectomy in symptomatic patients with high-grade carotid stenosis. N Engl J Med 1991;325:445-53.

14. O'Brien E, Mee F, Atkins N, et al. Evaluation of three devices for self-measurement of blood pressure according to the revised British Hypertension Society Protocol: the Omron HEM-705CP, Philips HP5332, and Nissei DS-175. Blood Press Monit 1996;1:55-61.

15. Bruno A, Shah N, Lin C, et al. Improving modified Rankin Scale assessment with a simplified questionnaire. Stroke 2010;41:1048-50.

16. Mahoney FI, Barthel DW. Functional evaluation: the barthel index. Md State Med J 1965;14:61-5.

17. ZUNG WW. A self-rating depression scale. Arch Gen Psychiatry 1965;12:63.

18. Dorman PJ, Slattery J, Farrell B, et al. A randomised comparison of the EuroQol and Short Form-36 after stroke. BMJ 1997;315:461.

19. Roccaforte WH, Burke WJ, Bayer BL, et al. Validation of a telephone version of the mini-mental state examination. J Am Geriatr Soc 1992;40:697-702.

20. Desmond DW, Tatemichi TK, Hanzawa L. The Telephone Interview for Cognitive Status (TICS): Reliability and validity in a stroke sample. Int $J$ Geriatr Psychiatry 1994;9:803-7.

21. Gray LJ, Ali M, Lyden PD, et al. Interconversion of the National Institutes of Health Stroke Scale and Scandinavian Stroke Scale in acute stroke. J Stroke Cerebrovasc Dis 2009;18:466-8.

22. Orrapin S, Rerkasem K. Carotid endarterectomy for symptomatic carotid stenosis. Cochrane Database Syst Rev 2017;6:CD001081.

23. Markus H, Cullinane M. Severely impaired cerebrovascular reactivity predicts stroke and TIA risk in patients with carotid artery stenosis and occlusion. Brain 2001;124:457-67.

24. van der Grond J, Balm R, Klijn CJ, et al. Cerebral metabolism of patients with stenosis of the internal carotid artery before and after endarterectomy. J Cereb Blood Flow Metab 1996;16:320-6.

25. Reinhard M, Schwarzer G, Briel M, et al. Cerebrovascular reactivity predicts stroke in high-grade carotid artery disease. Neurology 2014;83:1424-31.

26. Jusufovic M, Sandset EC, Bath PM, et al. Effects of blood pressure lowering in patients with acute ischemic stroke and carotid artery stenosis. Int J Stroke 2015;10:354-9.

27. Willmot M, Ghadami A, Whysall B, et al. Transdermal glyceryl trinitrate lowers blood pressure and maintains cerebral blood flow in recent stroke. Hypertension 2006;47:1209-15. 
28. Kate MP, Asdaghi N, Gioia L, et al. Blood pressure lowering with glyceryl trinitrate is not associated with improvement in cerebral perfusion. International Journal of Stroke 2015;10:77-438.

29. Morikawa E, Rosenblatt S, Moskowitz MA. L-arginine dilates rat pial arterioles by nitric oxide-dependent mechanisms and increases blood flow during focal cerebral ischaemia. Br J Pharmacol 1992;107:905-7.

30. Bath PM, Scutt P, Anderson CS, et al. Prehospital transdermal glyceryl trinitrate in patients with ultra-acute presumed stroke
(RIGHT-2): an ambulance-based, randomised, sham-controlled, blinded, phase 3 trial. The Lancet 2019.

31. European Carotid Surgery Trialists Collaborative Group. Randomised trial of endarterectomy for recently symptomatic carotid stenosis: final results of the MRC European Carotid Surgery Trial (ECST). Lancet 1998;351:1379-87.

32. Endovascular versus surgical treatment in patients with carotid stenosis in the carotid and vertebral artery transluminal angioplasty study (CAVATAS): a randomised trial. Lancet 2001;357:1729-37. 